Chapman University

Chapman University Digital Commons

ESI Publications

Economic Science Institute

8-10-2017

Human and Monkey Responses in a Symmetric Game of Conflict with Asymmetric Equilibria

Sarah F. Brosnan

Sara A. Price

Kelly Leverett

Laurent Prétôt

Michael Beran

See next page for additional authors

Follow this and additional works at: http://digitalcommons.chapman.edu/esi_pubs

Part of the Economic Theory Commons, and the Other Economics Commons 


\section{Human and Monkey Responses in a Symmetric Game of Conflict with Asymmetric Equilibria}

\section{Comments}

NOTICE: this is the author's version of a work that was accepted for publication in Journal of Economic Behavior \& Organization. Changes resulting from the publishing process, such as peer review, editing, corrections, structural formatting, and other quality control mechanisms may not be reflected in this document. Changes may have been made to this work since it was submitted for publication. A definitive version will be subsequently published in Journal of Economic Behavior \& Organization in 2017. DOI:

10.1016/j.jebo.2017.07.037

The Creative Commons license below applies only to this version of the article.

\section{Creative Commons License}

\section{(c) $10(9)$}

This work is licensed under a Creative Commons Attribution-Noncommercial-No Derivative Works 4.0 License.

\section{Copyright}

Elsevier

\section{Authors}

Sarah F. Brosnan, Sara A. Price, Kelly Leverett, Laurent Prétôt, Michael Beran, and Bart J. Wilson 


\section{Accepted Manuscript}

Title: Human and Monkey Responses in a Symmetric Game of Conflict with Asymmetric Equilibria

Authors: Sarah F. Brosnan, Sara A. Price, Kelly Leverett, Laurent Prétôt, Michael Beran, Bart J. Wilson

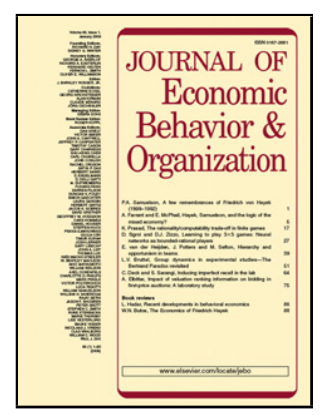

PII: $\quad$ S0167-2681(17)30215-9

DOI: $\quad$ http://dx.doi.org/doi:10.1016/j.jebo.2017.07.037

Reference: $\quad$ JEBO 4117

To appear in: Journal of Economic Behavior \& Organization

Received date: $\quad 24-3-2017$

Revised date: $\quad 22-7-2017$

Accepted date: $\quad$ 29-7-2017

Please cite this article as: Brosnan, Sarah F., Price, Sara A., Leverett, Kelly, Prétôt, Laurent, Beran, Michael, Wilson, Bart J., Human and Monkey Responses in a Symmetric Game of Conflict with Asymmetric Equilibria.Journal of Economic Behavior and Organization http://dx.doi.org/10.1016/j.jebo.2017.07.037

This is a PDF file of an unedited manuscript that has been accepted for publication. As a service to our customers we are providing this early version of the manuscript. The manuscript will undergo copyediting, typesetting, and review of the resulting proof before it is published in its final form. Please note that during the production process errors may be discovered which could affect the content, and all legal disclaimers that apply to the journal pertain. 


\title{
Human and Monkey Responses in a Symmetric Game of Conflict with Asymmetric Equilibria*
}

Sarah F. Brosnan, ${ }^{1,2}$ Sara A. Price, ${ }^{1}$ Kelly Leverett, ${ }^{1}$ Laurent Prétôt, ${ }^{1}$ Michael Beran, ${ }^{1}$ and Bart J. Wilson ${ }^{3}$

Corresponding Author:

Bart J. Wilson

Economic Science Institute

Smith Institute for Political Economy and Philosophy

Chapman University

Orange, CA 92866

Email: bartwilson@gmail.com

Phone: 714-628-7306

* This research was funded by NSF SES 1123897 to SFB, MJB, BJW, and Steven J. Schapiro and NSF SES 1123803 to BJW. We thank the editor and two anonymous reviewers for comments that have improved the paper, Megan Luetje for recruiting our human participants, and Jeffrey Kirchner for his software programming.

${ }^{1}$ Georgia State University, Language Research Center and Department of Psychology

${ }^{2}$ Georgia State University, Neuroscience Institute and Department of Philosophy

${ }^{3}$ Chapman University, Economic Science Institute and Smith Institute for Political Economy and Philosophy

\section{Highlights}

- We compare human and monkey responses in a symmetric game of conflict.

- Both rhesus macaques and capuchins played a Nash equilibrium in asynchronous play.

- Humans were the only species to play both asymmetric equilibria in a repeated game.

- These results suggest that multiple cognitive mechanisms underlie decision-making.

\begin{abstract}
To better understand the evolutionary history of human decision-making, we compare human behavior to that of two monkey species in a symmetric game of conflict with two asymmetric equilibria. While all of these species routinely make decisions in the context of social cooperation and competition, they have different socio-ecologies, which leads to different predictions about how they will respond. Our prediction was that anti-matching would be more difficult than matching in a symmetric coordination with simultaneous moves. To our surprise, not only do rhesus macaques frequently play one asymmetric Nash equilibrium, but so do capuchin monkeys, whose play in the coordination game was literally not distinguishable from randomness (in simultaneous play). Humans are the only species to play both asymmetric equilibria in a repeated game.
\end{abstract}

Keywords: coordination, Hawk-Dove, chicken game, capuchin monkeys, rhesus monkeys, experimental economics 


\section{INTRODUCTION}

Why do humans make the decisions that they do? One way to address this is to compare our decision-making with that of our relatives in the animal kingdom. How are our decisions similar, and in what ways do they differ? How did our decision-making evolve, both in terms of what constrained it and what motivated it during its phylogenetic development? What is its evolutionary function? Several researchers have taken this approach, comparing humans' responses to those of other primates in an attempt to understand the evolutionary trajectory of our decision-making (Brosnan et al. 2013; Chen et al. 2006; Santos and Rosati 2015). By studying other species, we learn about the evolutionary history of our own behaviors, which highlights behavior and cognitive processes that are unique to humans and those that are shared more broadly. This can help us understand the evolutionary pressures that early humans were under, as well as highlighting the constraints under which human decision-making evolved. Moreover, by looking at a variety of species that inhabit different socio-ecological niches, we can begin to understand how these different social and ecological factors may have influenced the evolution of decision-making. Second, other species can be a good model system of humans, allowing us to ask questions that are difficult or impossible using humans. For example, other species can be tested repeatedly over many years, in highly controlled circumstances, with individuals for whom we know their relationships and previous experience. This allows us to study other species with a level of detail and precision that is impossible in humans, and may uncover important contexts that influence decisionmaking.

These sorts of comparative studies require close attention to detail. In order to tell whether differences among species are due to differences in the species' behaviors or differences in procedures it is necessary to have procedures that are as similar as possible, otherwise purported differences between humans and other species may turn out to be differences in procedure, making it impossible to determine continuities in decision-making behavior (i.e., Jensen et al. 2007; Smith and Silberberg 2010; likewise, similarities based on different procedures may not indicate actual similarities among species). In previous comparative studies of cognition, humans have typically had a large advantage in terms of being provided verbal instructions, payoff matrices, and pre-tests to verify understanding. To tackle this problem, we have developed a research program in "comparative experimental economics," using the methods of experimental economics to compare species across the Primate order (Brosnan et al. 2013). The utility of experimental economics for comparative work lies in the approach, which breaks complex decisionmaking into very simple, often dichotomous choice tasks that can easily be instantiated across a variety of species in ways that do not require instruction or pre-testing. By designing the procedures around animals (eliminating the verbal component and not providing any a priori information) and then transferring these procedures to humans, we can level the playing field and more accurately test for species differences. This allows us to directly compare outcomes and, therefore, provide a much more nuanced understanding of how human decision-making relates to that of other primates.

Such a comparison is complex, and therefore requires that we break the question of animal decision-making down into multiple components. Indeed, our first goal has been simply to document the degree to which humans and other species reach - or do not reach - similar outcomes when given similar decision-making scenarios. Note that this is a purely outcome-based assessment, without reference to the underlying mechanisms leading to these decisions. In this way, we are looking for the behavior's pattern and spread, with the goal of understanding function. If, for example, all species show the same decision-making patterns, then we have evidence that this behavior is so critical to primates that the functional outcome occurs across different species (whether or not the underlying mechanism is the same). On the other hand, an outcome that occurs only in humans may indicate that the behavior requires 
too great of a cognitive load, or that the decision situation is not one in which animals routinely find themselves, so that there has been no pressure for a behavioral solution to evolve.

A second step, on which we have not yet embarked, is to explore the mechanism by which subjects make the decisions that they do. In particular, this includes subjects' underlying motivations, or what they intended to do. Do subjects understand the decision scenario, or are they just engaging in a set of stereotyped behaviors that have been selected because of the benefit that they provide? What sort of memory, individual recognition, planning, etc are required for a given behavior? If there are similarities in outcome, to what degree are the underlying mechanisms also similar? Do similar mechanisms lead to different outcomes in different species or contexts? This second step is tricky; unlike in humans, we cannot ask other species to explain why they made a decision that they did. But more insidiously, motivations are also surprisingly difficult to study in humans, who may not know their own motivation, or may intentionally or accidentally report false motivations [such as in the social desirability bias (Crowne and Marlowe, 1960)]. As a result, we think it is worthwhile to begin at the level of behavioral outcomes with all species, which provides information on what subjects actually do, before attempting to uncover their motivations.

Our original work explored whether four species of primate played coordinated outcomes in a symmetric coordination game, the assurance game, which requires individuals to make the same choice in order to achieve the payoff-dominant outcome (Brosnan et al. 2011; Brosnan et al. 2012). For this work, subjects chose one of two strategies (Stag or Hare), by either choosing one of two tokens or one of two icons on a computer screen. The outcomes of those choices were dependent upon what both players chose. Coordination on Stag yielded the greatest payoff (4 rewards for each player) while choosing Hare was the risk dominant outcome (1 reward no matter what the partner chose; choosing Stag when one's partner chose Hare resulted in no payout. See Figure 1a for the payoff matrix). In a decision situation such as this, subjects who recognize that their payoffs are tied to their outcomes should make a consistent set of responses; on the other hand, subjects who do not understand this should show patterns of play that are not statistically distinguishable from random play. Moreover, when there is an clearly better outcome [here the payoff dominant (Stag, Stag) outcome], if subjects fail to achieve it we need to explore what led to this non-optimal outcome, especially if there is variability across species.

We chose the four species that we did because they are highly social species that, across different contexts, cooperate with non-kin [humans, chimpanzees, rhesus monkeys, and capuchin monkeys: Kappeler and Van Schaik (2005); Brosnan (2010); Muller and Mitani (2005); Maestripieri (2007)]. For instance, all four species form extensive coalitions and alliances, humans and chimpanzees cooperatively hunt, and the apes and capuchin monkeys cooperatively defend group boundaries. Only humans and chimpanzees show signs of actively coordinating, with different individuals taking different roles. Aside from these differences in the form and function of cooperative interactions, these species also live in very different ecologies and have very different social structures, which will also influence their behavior. Capuchins live in very small, cohesive social groups of 10-25 individuals, while the other three species may live in groups of more than 100. Additionally, chimpanzees live in a fission-fusion social structure in which some pairs of individuals may not see each other for many days. Rhesus monkeys stand out for being more despotic and having more linear hierarchy than the other three species. This despotism is likely what precludes many of the forms of cooperation seen in the other species, although again, they do exhibit coalitions and alliances (typically in an aggressive context: Pirta 1983, Higham and Maestripieri, 2010). While four species is not sufficient to make conclusive predictions about the influence of socioecology, we can begin to extrapolate how differences in choices in this game correlate with these factors so as to make predictions for future work. 
We found that at least some pairs in all four species played the coordinated, payoff-dominant outcome of Stag-Stag, but how they did so varied. Chimpanzees not only played the payoff dominant Nash equilibrium, but quickly found it a second time when presented with novel tokens that were rewarded using the same payoff matrix. This indicates that they comprehended the underlying strategy of the game, rather than relying on a propensity to choose one token over the other. Interestingly, however, chimpanzees showed a strong experience effect, with subjects who had extensive experience with cognitive tasks finding the payoff dominant Nash equilibrium whereas those who had very little such experience did not (Brosnan et al. 2011). In general, chimpanzees are capable of high levels of coordination even when coordination partners are spatially separated, performing different roles, and out of view of one another (i.e., group hunting; Boesch, 1994), so it is not necessarily surprising that they do so well in this task.

Our Old World monkey species, rhesus monkeys, played the payoff dominant Nash equilibrium with simultaneous moves as well as did humans, even when they could not see what their partner played. Rhesus groups are very large, sometimes numbering hundreds of individuals, which may have strongly selected for the ability to remember and extrapolate from past interactions. A follow-up study in which subjects played a computer simulated partner demonstrated that humans and rhesus used different mechanisms. Rhesus showed a consistent propensity to play Stag across all patterns of random play ( $0 \%$, $25 \%, 50 \%, 75 \%$, and $100 \%$ Stag) whereas humans matched the frequency with which the simulation played Stag (Parrish et al. 2014). Thus, the evidence indicates that rhesus were selected to be able to coordinate, but using a different, and presumably simpler, mechanism than is seen in humans.

Capuchin monkeys, our New World monkey species, were unable to settle on either Nash equilibrium with simultaneous play (their play was not distinguishable from random choices, indicating that they did not understand that their choices influenced the outcomes). However, with (endogenous) sequential play could they coordinate on the payoff dominant Nash equilibrium. This indicated that capuchins were using matching, presumably in combination with a propensity to choose the sometimes higher-paying Stag option, to solve the task (Brosnan et al. 2011; Brosnan et al. 2012). While capuchins are highly cooperative, the fact that they live in small, cohesive social groups and are rarely out of sight of one another may have meant that there was little selective pressure for the ability to remember outcomes that are not directly witnessed. Such a mechanism would be more than sufficient for cooperative endeavors in which subjects are in view of one another almost continuously.

Of course, what is important is to understand the interplay between species' socio-ecologies and the cognitive mechanisms that they have available. Our previous work on the indicated that each species used a different mechanism, but it is important to see the degree to which these explanations and the posited underlying mechanisms can explain decision-making in other contexts. It is one thing to find a Nash equilibrium in a symmetric coordinated game, but how do other species perform relative to humans in a different game with conflict between the two players? For the current study, we expanded this line of research to a symmetric game of conflict with asymmetric equilibria. Our anti-coordination game is similar to the classic game introduced by Maynard Smith and Price (1973) and later named the HawkDove game (Maynard Smith 1982), in which two organisms compete over a shared resource. See Figure $1 \mathrm{~b}$ for our payoff matrix with two strategies, Fight or Yield. The game with two asymmetric Nash equilibria [(Fight, Yield) and (Yield, Fight)] is such that each partner has a temptation to choose Fight, as the highest payoff in a one shot game is only possible by playing this strategy. In the one shot game both would prefer the Nash equilibrium in each ' $I$ ' fight and 'you' yield. The question is, who fights and who yields? 
We studied three of the previously-studied species, capuchin monkeys (Cebus [Sapajus] apella), rhesus monkeys (Macaca mulatta) and humans (Homo sapiens). These species were chosen because, whereas all species in previous work played the payoff dominant Nash equilibrium in the assurance game task (with endogenous sequential moves), the non-human species did so by matching (capuchins) or preferring (rhesus) the maximizing Stag token, which is not efficacious in our anti-coordination task. This allowed us to explicitly test whether subjects could use alternate approaches, and whether our proposed explanations based on socio-ecology continued to explain our results.

We used two different versions of our game of conflict to make sure that if subjects played randomly, it was not because we failed to present it in a way that they comprehended. ${ }^{1}$ This is essential as often studies that were designed to look at the same behavior produce very different results, presumably indicating that the primates perceived them differently than humans do, or at least than the humans had intended (e.g., studies of prosocial behavior; Claidière et al. 2015; Horner et al. 2011; House et al. 2014; Jensen et al. 2006; Silk et al. 2005). Capuchins were given 1) a manual version of the task, in which they chose between two tokens, one representing Fight and the other Yield and 2) a computerized version of the task, in which they chose between two on-screen icons, one representing Fight and the other Yield. There were two versions of these tasks, one in which the partner's choices were immediately visible (the asynchronous or endogenous sequential task) and one in which both players' choices were hidden until they had both made a decision (a functionally simultaneous task). The rhesus monkeys did not participate in the manual version of the task because those monkeys were only accustomed to computerized tasks (which gives manual tasks limited validity) and because previous work in the assurance game and the current results both showed that capuchin monkeys' choices did not differ between the computerized and manual versions of the task.

In general, we predicted that capuchin monkeys would not play either Nash equilibrium, whether or not they could see their partner's play. Only with sequential play could the capuchins coordinate on the payoff dominant Nash equilibrium in the assurance game. Because matching the token is inefficient in our game of conflict, we predicted that they would be unable to "anti-match," which is cognitively more difficult than matching (Martin et al. 2014). It was important, given this prediction, to test the capuchins on every experimental version of the game that we designed to make sure that this was a general finding and not limited to a specific testing modality.

We did not have a prediction for the rhesus monkeys. Our work with the assurance game indicated that they found the payoff dominant Nash equilibrium with a simple propensity to choose the more valuable Stag option (Parrish et al. 2014), but this would be unstable and inefficient whether they both played Fight or Yield in our game of conflict. Other work, however, shows that rhesus outperform capuchins on at least some complex cognitive tasks that require them to monitor outcomes (e.g., metacognition tasks: Beran and Smith 2011; Beran et al. 2009), leaving open the possibility that they would be able to settle on at least one efficient Nash equilibria using a mechanism that wasn't apparent in the previous work. Unlike capuchins, whose play in a simultaneous assurance game literally cannot be distinguished from pure randomness, we predicted that the rhesus, as in the assurance game, would be more likely to develop a consistent pattern of play, whatever it might be.

We additionally predicted that humans would find a Nash equilibrium or both Nash equilibria, but how would this play out in repeated trials? Would pairs settle on one Nash equilibrium or the other, or

\footnotetext{
${ }^{1}$ We also tried a third version of the task that the primates apparently failed to understand. These results are included in Appendix A.
} 
would they alternate play between the two Nash equilibria? We further predicted that, as in the assurance game, there would be no difference between the computerized and manual tasks for the humans.

\section{General Methods}

Parameterization

Notice that in both the assurance game and our conflict game, the highest and lowest possible payoffs are the same, 4 and 0 , respectively (see Figure 1 ). This is by design. The decision to Fight will result in a payoff of 4 or 0 , just like the decision to the play Stag in the assurance game. The only new payoff possibility in the conflict game is 2 , when both players Yield. To make the difference more salient between the Nash equilibrium Fight payoff and the (Yield, Yield) payoff, we chose $(2,2)$ over $(3,3)$ for the (Yield, Yield) payoff. (Fractional payoffs are not possible with the reward dispenser, nor is it clear how nonhuman primates would perceive them.) Thus this means that the decision to Yield will result in a payoff of 2 or 1 , which is only slightly different than playing Hare for a sure payoff of 1 . In both the assurance game and our conflict game, there is a high spread in payoffs for one strategy and a low/zero spread in payoffs for the other strategy. The key difference, of course, is that the assurance game is a coordination game and our conflict game an anti-coordination game.

\section{Equivalent Procedures across Species}

We implemented procedures that are as similar as possible across species in nearly equivalent environments, which is critical in order to be able to compare species' responses (Brosnan et al. 2011). In keeping with this, no subject received verbal instruction on the game. Because humans expect some communication from the experimenter, the monitor made the following brief remarks before the experiment began:

1) Have you participated in an economic experiment before? (Both were screened at recruitment to reply with a "yes.")

2) In this experiment you will be using red and blue chips to make decisions behind a partition.

3) As the experiment progresses you may be paid in quarters or dollar bills.

4) Please collect the coins in the yellow cups provided.

5) These are the only instructions you will receive in the experiment. Once the experiment begins, the experimenter will not be allowed to answer any questions until the experiment is over.

6) Do you have any questions before the experiment begins?

All species had the capability to communicate with their partner in whatever way possible for that species (speaking, vocalizations, gestures, body posture, facial expressions, etc.).

Additionally, no species was shown the payoff matrix, nor did any species receive training, previous experience with this task and payoff matrix, or pre-tests to assess understanding, so all participants had to discover the payoff structure during the course of the game (all nonhuman primates easily perform similar kinds of tasks with varied response strategies that must be retained in memory, such as in our previous work). All subjects were rewarded on each trial rather than accumulating rewards and being paid at the end.

Finally, and again differently from the majority of human studies, no subject was tested anonymously; all subjects sat directly adjacent to their partner, which is common in nonhuman primate testing but not in human testing. Therefore, as mentioned previously, subjects could communicate with one another. Non-human primates were tested with members of their social group whereas humans, 
while strangers, were all students at the same university, which presumably also instituted a minimal "in group" mentality.

\section{Food Magnitude Pre-testing}

Prior to the study, we verified that all monkey subjects preferred greater numbers of reward pellets over less numbers to ensure that their choices were made with the goal of achieving the maximum number of rewards. Subjects should be able to do this for all quantities used in our study (Addessi et al. 2008; Beran et al. 2008; Evans et al. 2009; Washburn and Rumbaugh 1991), nonetheless subjects were tested in every ratio used in the study to verify that all preferred the greater quantity at least $80 \%$ of the time (all subjects tested passed this pre-test). We assumed that all human subjects preferred to receive more money to less money.

\section{Human Subjects}

We tested undergraduate subjects from Chapman University, Orange, CA ( $n=52$ for manual task and $n=54$ for computerized task). Students were recruited via an email system and paid $\$ 7$ for showing up on time, plus what they earned in the experiment. Each subject had participated in at least one economic experiment before, as all capuchins had previous experience making decisions during experiments to receive food rewards. Subjects were, however, excluded if they had previously participated in a game similar to our conflict game structure (i.e., a Hawk-Dove or Chicken game) or any other normal form game. Subjects only participated in one of the below studies, never both.

\section{Capuchin Subjects}

We tested four pairs of adult (and one sub-adult) capuchin monkeys ( $n=8,5$ male) for the manual task and five pairs of capuchin monkeys ( $n=10,6$ male) in the computerized tasks. Pairs remained the same throughout the study and four pairs participated in both the manual and computer tasks. Monkeys were socially housed at the Language Research Center of Georgia State University in mixed sex social groups with extensive indoor and outdoor living space complete with environmental enrichment. Subjects voluntarily separated into attached individual cages for testing. Subjects were never food or waterdeprived and water was available at all times, including during testing. Subjects received pellets as rewards, in addition to their daily diet of primate chow, fruits, vegetables, and other enrichment.

\section{Rhesus Subjects}

We tested two pairs of adult male rhesus monkeys $(n=4)$ on the computerized tasks. Pairs remained the same throughout the study. Monkeys were housed at the Language Research Center of Georgia State University. Monkeys lived in individual cages in rooms with other monkeys with whom they were socially compatible and with whom they shared outdoor time. Subjects were never food or waterdeprived and water was available at all times, including during testing. Subjects received pellets as rewards, in addition to their daily diet of primate chow, fruits, vegetables, and other enrichment. For testing, subjects were moved from their home area to our paired testing room, with which they were familiar from previous work (Brosnan et al. 2012) and which they only used for tasks in which they were paired with another rhesus monkey.

\section{STUDY 1: Manual task}

\section{Study 1 Methods}

Each subject was provided with two token choices, one representing Fight and one representing Yield (see details for each species below). Humans' tokens were poker chips and capuchin monkeys' tokens were polyvinyl chloride (PVC) pipes $20 \mathrm{~cm}$ in length and $1.9 \mathrm{~cm}$ in diameter (capuchin tokens were 
patterned due to the fact that many capuchins are dichromats and may have difficulty discriminating some colors; Gomes et al. 2002; Jacobs 2007). After choosing a token, subjects returned the chosen token to the experimenter, who then held up both tokens, followed by both rewards, then presented each subject with their respective reward (see Figure $1 \mathrm{~b}$ for the payoff matrix used for all studies). Since no subject of any species received pre-training, subjects not only had to learn the payoffs during the course of testing, but also had to learn to return only a single token. For all species, if subjects initially returned more than one token, the experimenter returned all tokens to the subject and allowed them to try again. At such a point as the subject offered a single token, the experimenter accepted it immediately. Note that all capuchin monkeys were familiar with exchanging tokens with human experimenters prior to the start of the study (e.g., Brosnan et al. 2011). Below we discuss the details of each species' specific procedures.

\section{Capuchins}

Subjects sat in separate halves of a joint box (24" tall, 56" long, 27.5" wide) attached to their home enclosures. Two experimenters were present, each trading with one subject. Capuchins were rewarded with banana flavored pellets that were presented in colored/patterned cups (the color/pattern based on the number of rewards) to provide an additional cue to the number of rewards that were given to each monkey. This was done to emphasize to each subject how many rewards they got and how many their partner got (we were worried that they would have trouble determining how many pellets their partner received without this additional cue).

Capuchins were tested in two conditions: opaque barrier and clear barrier. In the opaque barrier version, a divider with an opaque lower section separated subject and partner, allowing monkeys to see each other's faces but not their tokens. Thus, in this condition subjects' play was functionally simultaneous because they did not know what their partner chose prior to making their own choice. In the clear barrier version, a clear divider was used, allowing monkeys to potentially see their partner's token choice before returning their token (although note that we do not know whether they looked at their partner's play, or made use of this information if they did so; due to the lack of sclera and prominent brow ridges that shade the eyes, identifying where NHPs look without an eye-tracker is unreliable). We began with the opaque barrier to see if subjects could settle on the Nash equilibrium in the more difficult situation, in which they could not see their partner's choices. We did not counterbalance because it was important to test this functionally simultaneous version first so that we could better interpret the results. If they played the sequential version first and played the $\mathrm{NE}$, we would then have been unable to tell whether the results on the simultaneous version were because the understood it, or were simply repeating the pattern of play that they had developed (although note that they were unable to find the NE in the simultaneous version of our previous Assurance Game work even after having found it in the sequential version). When they did not play the NE, we next tested them in the clear barrier condition. Pairs first received 10 sessions of 40 trials each with an opaque barrier, and then 10 additional sessions of 40 trials each with the clear barrier.

\section{Humans}

Upon hearing the instructions, pairs were seated in desk chairs next to one another in front of a circular table. We used a T-shaped partition that horizontally separated the experimenters from the subjects (much as the caging did in the monkey studies; this was the same set-up used in Brosnan et al. 2011). The partition allowed for eye contact between subjects. Subjects could see each other's choices if they looked around the partition, but there is no recorded evidence that they did so. The barrier had a 2" $x$ 5.5" slot at the bottom of each side through which the experimenter could slide the two tokens at the 
beginning of each trial. Subjects returned a single token through a 1" $\times 5$ " slot about half-way up the partition, dropping it into a ceramic bowl so that their partner could hear when they had made a choice. Subject and partner were rewarded after each trial (in quarters or a single dollar bill). Humans participated a single session of 40 trials.

\section{$\underline{\text { Results }}$}

\section{Capuchins}

Figure 3 reports the proportion of Fight play by pair in both the opaque and clear barrier conditions. The clumping of the data in the middle displays the failure to reject the null hypothesis of random play by the subjects.

\section{Stage 1: Opaque barrier condition}

The opaque barrier condition, in which subjects could not see their partner's play, was presented first to all subjects. None of the capuchin pairs' choices deviated from chance across sessions. (One pair alternated playing the two Nash equilibria in the final session $\left(\chi^{2}=4.95, \mathrm{df}=1, p<0.05\right.$, but given how many sessions there were overall, this could be due to chance).

\section{Stage 2: Clear barrier condition}

In the clear barrier condition, when able to see partner's token choice, two pairs alternated playing the two Nash equilibria within a single session $\left(\chi^{2}>4.0, \mathrm{df}=1, p<0.05\right)$, but this behavior was not maintained across sessions. Individual monkeys' strategies were not statistically different from random; neither subject nor partner showed a propensity to play Fight or Yield (binomial test; all $p$-values $>0.05$ ) and all players had an expected number of runs (all $p$-values $>0.05$ ).

\section{Humans}

Eleven out of 26 human pairs deviated from chance; nine pairs of these 11 pairs played Nash equilibria, alternating Fight and Yield (all $\chi 2>14.83, \mathrm{df}=1$, all $p$-values $<0.001$ ), one pair played the mutually destructive (Fight, Fight) ( $p<0.001$, Fisher's exact test) and one pair played (Fight, Yield), but without alternating between partners ( $p<0.05$, Fisher's exact test). ${ }^{2}$ For all 9 human pairs that alternated, individuals' behavior within pairs showed the same patterns. Neither player exhibited a propensity to choose Fight or Yield (binomial tests, all $p$-values $>0.05$ ), and in each pair, both players had a greater than expected number of runs (i.e., sequential plays of the same option; runs test, all $p$-values $<0.05$, ). Therefore, our evidence indicates that in all 9 pairs, both individuals alternated between strategy choices at an equally high rate.

\section{Discussion}

Approximately one third of human subjects played alternating Nash equilibria while none of the capuchins did, whether or not they could see their partner's play. This differs from their behavior in the Assurance Game, in which capuchins performed as well as humans when they could see their partner's choices. This supports our hypothesis that the matching strategy that capuchins used in the assurance game, while sufficient to find the payoff dominant outcome in that game, was insufficient for the more complex Conflict Game. We note that some pairs did find the alternating solution during a single session, but were unable to maintain the strategy across sessions. Although this could be due to chance, it was the only non-random play we saw and thus may indicate that with additional experience, subjects can learn the task, which we test in Study 2.

\footnotetext{
${ }^{2}$ Because humans tend to alternate play between the two Nash equilibria, the analogous figures for humans are reported in Appendix B.
} 
In contrast to the capuchins, humans played alternating Nash equilibria. In pairs that did alternate play between the two asymmetric Nash equilibria, individuals followed statistically non-random strategies (e.g., alternating between choices at a higher rate than expected by chance, and showing no preference for one strategy over the other), suggesting that individuals may have understood their choices as part of a larger strategy. In comparison, individual monkeys' strategies were not statistically different from random, even in sessions in which they played alternating Nash equilibria. Of course, not all human pairs played alternating Nash equilibria, although we note that despite the fact that this task is more difficult to solve than the assurance game, approximately the same percentage of pairs found the payoff dominant outcome as in the assurance game (Brosnan et al. 2011). Finally, one human pair settled on non-stage game Nash equilibria (Fight, Fight) and one settled on non-alternating Nash equilibrium (Fight, Yield). The frequency of these other strategies was too low for statistical analysis, but this outcome is worth exploring in future studies.

\section{STUDY 2: Computerized task}

\section{Study 2 Methods}

This study was designed to replicate Study 1 using a computerized procedure. This was done in order to maximize the opportunities for subjects to excel in the task. Although previous work showed that capuchin monkeys and humans both performed equally well on manual and computerized versions of the Assurance Game (Brosnan et al. 2011; Brosnan et al. 2012), we wanted to validate that this was also the case in other games with different payoff structures. The payoff matrix was identical, as was the methodology, with the exceptions that were essential to move it to the computer, detailed below.

Subjects made their decision by choosing one of two icons on each side of a split computer screen, one representing Fight and the other representing Yield. At the start of each trial, both icons were presented in a vertical distribution; the order of presentation was randomized across trials and across individuals within a single trial. Each subject controlled a cursor via a joystick. There were no constraints on order of play or the timing of decisions. Once both subjects had made a decision, each subject received (or not) rewards depending on the combination of what they chose and what their partner chose, following the same payoff matrix used in Study 1 (see Figure $1 \mathrm{~b}$ ).

As in Study 1 and our previous work on the assurance game study, there were two versions of the task: asynchronous and functionally simultaneous. In the asynchronous version, decisions were revealed as they were made, and individuals could potentially use this information when they made their own decision. Again, subjects' order of play was never constrained, nor was there a time-out period, so in principle choices could still be made at the same time. Additionally, even if the choices were sequential we do not know if they looked at their partner's choice, or used that information. In the functionally simultaneous version, when an individual made a choice, their side of the screen went blank, but their choice was not displayed until their partner had also made their choice, at which point both icons were displayed simultaneously. Moreover, the cursor did not move and the joysticks were hidden, so there was no way for the monkeys to use their partner's or their partner's cursor's movements to see or to infer their partner's choice. Therefore, subjects did not know what their partner had chosen until after both choices were completed. Capuchin monkeys were tested first in the asynchronous version, since we knew from Study 1 that they were unlikely to be able to solve the more challenging functionally simultaneous version. As in the assurance game, humans were first tested in the functionally simultaneous version and, given their results, were not tested on the asynchronous version. 


\section{Capuchins}

We tested monkeys in adjacent individual testing boxes, allowing the monkeys clear visual access to both their own and their partner's side of the computer screen. Figure 3 displays two photographs of the testing apparatus. The computer rested on a moveable cart; a large screen was placed at the monkeys' eye-level. Joysticks and automatic reward dispensers were connected to the computer on either side of the cart. Computerized test sessions involved 10 sessions of up to 200 trials (pairs could complete as many trial blocks as they chose, up to 200 total trials, within a two-hour time frame). Monkeys were tested in 3 stages. In this case, because they had been unable to find the NE in the previous Study, we began with the easier sequential version. Stage 1 was the asynchronous version of the task. Given the fact that all five pairs developed the (Fight, Yield) strategy, we repeated the same task using different icons in order to see if this was a chance outcome or a consistent behavior, and to determine whether the same individuals consistently played the same strategy (Stage 2). Stage 3 used the same icons as in Stage 2 , but was functionally simultaneous. This was to see whether they were able to continue the NE play in the simultaneous version once they had found it in the sequential version.

\section{Rhesus}

We tested four adult male rhesus monkeys in a specially designed paired testing area used in the previous study (Brosnan et al. 2012; Parrish et al. 2014). All features of the procedure were the same as those described for the capuchins, except that due to testing constraints the rhesus monkeys were given only 5 sessions each of Stage 1 and Stage 2. Computerized test sessions of each stage involved 5 sessions of up to 200 trials (pairs could complete as many trials as they chose, up to 200 total trials, within a twohour time frame). As with the capuchins in Study 1, all subjects began with the simultaneous version. In previous work using the assurance game, rhesus monkeys played the payoff-dominant NE in both the simultaneous and sequential versions, so it was important to test them first on the simultaneous version. Note that this meant that both rhesus and capuchins had had previous experience with the game, albeit in different contexts, prior to the sequential computerized task, making their results more comparable.

\section{Humans}

Each session consisted of one pair of participants, who were the only two individuals in the room. The participants were seated next to one another at a single computer on the same table used for the exchange task above. Instead of a mouse, the participants used joysticks that were concealed inside a wooden box to shield their hand movements and avoid cuing their partner. No participants received instruction on the task or the payoffs. Like with the poker chip task, they received the following brief remarks:

1) Have you participated in an economic experiment before? (Both were screened at recruitment to reply with a "yes.")

2) In this experiment you will be making decisions using a joystick attached to a computer. Use the left thumb pad to make a decision.

3) As the experiment progresses you may be paid in quarters by the machines next to your computer.

4) Please collect the coins in the yellow cups provided so as to not clog up the machines.

5) These are the only instructions you will receive in the experiment. Once the experiment begins, the experimenter will not be allowed to answer any questions until the experiment is over.

6) Do you have any questions before the experiment begins?

The cursor that appeared in the center of the screen never moved. A trial consisted of simply moving a joystick up or down in the direction of a red circle or light blue rectangle. The position of the symbols was randomly set each trial for each participant. After a symbol was chosen, both symbols disappeared from the screen. Once both subjects had made a choice, each chosen option was displayed 
in the center of that subject's half of the screen. During this time, the participants received payment in quarters from a coin dispenser, an analogue to the primates' pellet dispenser. The payoffs were in the same ratios as those of the monkeys. A session consisted of 40 trials.

\section{Results}

\section{Capuchins}

Figure 4 reports the proportion of Fight play by pair in these treatment conditions. The clumping of the data in the upper left and lower right corners displays the propensity to play a single asymmetric equilibrium.

\section{Stage 1: Original stimuli/asynchronous (sequential)}

There was a high occurrence of cells with zero values (meaning that combination of strategies was never played), making chi-square and Fisher's exact tests impossible. Thus, as with the exchange task we considered a strategy to be meaningful if the pair showed a $75 \%$ or greater preference for one of the four options (chance is 25\%; this is the same criterion used previously in the Assurance game, see Brosnan et al. 2012). In all pairs, one subject consistently selected Fight while the other consistently selected Yield. Pair 1 met criterion on 9/10 sessions, pairs 2 and 3 on 10/10 sessions, pair 4 on 9/10 sessions and pair 5 on $7 / 10$ sessions.

\section{Stage 2: New stimuli/ asynchronous (sequential)}

Using the same criteria of $75 \%$ or greater preference for one of the four outcomes, all five pairs again had a significant preference for a non-alternating (Fight, Yield) outcome, but in this case it was the opposite individual who played Fight for all five pairs. That is, the individual who played Fight in Stage 1 played Yield in Stage 2 in all five cases, indicating that this is not a result of dominance. Pair 1 met criterion on $4 / 10$ sessions, pairs 2,3 , and 4 on $8 / 10$ sessions, and pair 5 on $6 / 10$ sessions.

\section{Stage 3: New stimuli/functionally simultaneous}

When they could not see their partners' choice, these pairs played the four outcomes more evenly. Despite the fact that we used the same stimuli as in Stage 2, using the same criteria as in Stages 1 and 2, only Pair 5 had a slight propensity to play (Fight, Yield) in 6 of 10 sessions. Other than this, individuals showed no significant individual propensity to play Fight or Yield (all $p$-values $>0.05$ ), nor did they deviate from the expected number of runs in more than 2 or 3 sessions out of 50 in each Stage, all of which suggest that their play was random.

\section{Rhesus}

Figure 5 reports the proportion of Fight play by pair in these treatment conditions. The data in the upper left and lower right corners display the propensity to play a single asymmetric equilibrium by pair.

\section{Stage 1: Functionally Simultaneous}

Due to the small number of pairs, all rhesus monkeys were first tested on the synchronous task (if they were first tested on the asynchronous version it would be impossible to tell whether success in the presumably more difficult synchronous task was due to experience or not). Few cells had values of zero, so to determine whether they had significant preferences for one of the four outcomes, we used chi-square tests or, when there were cells with fewer than 5 observations, Fisher's exact tests. Both pairs showed a non-significant tendency towards a consistent pattern of play overall, although in neither case did they ever show a significant pattern on any given session. One pair tended to play (Yield, Fight) (Han/Obi: $p=0.071$, all individual sessions NS), although this was largely due to one subject that had a 
strong propensity to play Fight (Obi: binomial test $p<0.001$ in sessions $2-5$, but $p<0.001$ for Yield in session 1 ) while the other preferred Yield across all sessions (Han: binomial test all $p$-values $<0.001$ ). The other pair tended to avoid (Fight, Fight) (Luke/Chewie: $p=0.069$, all individual sessions NS), which was due to one subject consistently playing Yield (Chewie: binomial test, all $p$-values $<0.001$ ) while the other played Yield on the first 2 sessions (Luke, session 1: $p<0.001$, session 2: $p=0.030$ ), showed a tendency in that direction on the third $(p=0.060)$, and played Fight on the last two sessions (session 4: $p=0.044$, session 5: $p=0.021$ ). In most cases subjects' number of runs were no different than expected by chance, although there were a few sessions in which there were significantly more or fewer runs than expected. ${ }^{3}$

\section{Stage 2: Asynchronous}

In the asynchronous condition, only one pair (Luke/Chewie) significantly preferred the (Yield, Fight) strategy ( $p=0.0216)$; however as with the capuchin monkeys, there was a high occurrence of zero cells (or cells whose value is less than 5). Using the same criterion as the capuchins, both pairs preferred the (Yield, Fight) outcome (92.5\% of all trials for Han/Obi and $88.2 \%$ for Luke/Chewie). Individually, each monkey in both pairs showed a strong preference for one of the two outcomes (binomial tests: all $p$-values $<0.0001$ ). No pattern emerged with respect to the runs test, with each subject showing significant deviations from chance in some sessions, but not others (see data appendix for details).

\section{Humans}

Twelve out of 27 human pairs deviated from chance; eleven pairs alternated between the two asymmetric Nash equilibria (all $\chi^{2}>5.28, \mathrm{df}=1$, all $p$-values $<0.05$ ), and one pair played the mutually destructive outcome (Fight, Fight) ( $p<0.01$, Fisher's exact test). For eight of the eleven pairs that alternated between Nash equilibria, both players had a greater than expected number of runs $(p<0.05$, runs test). There were no observable sex differences in terms of pairs or individual play.

\section{Discussion}

Contrary to our predictions, and the results from Study 1, capuchin monkeys played one asymmetric Nash equilibrium in the computerized task with sequential play. Unlike in the manual task, in which no patterns of choice were evident, all five pairs in the current study showed a strong propensity to choose one of the two icons that led to consistent (Fight, Yield) play in both stages with asynchronous play (Stages 1 and 2). We did not expect the capuchins to be able to settle on a Nash equilibrium because it cannot be done via matching, and anti-matching is more challenging than matching (as evidenced by longer reaction times; Martin et al. 2014). We do not have a good explanation for how the monkeys developed this consistent patterning. One possibility is that they simply played the outcome that maximized their outcomes (i.e., if one monkey was already playing Fight, the second would not get rewarded for Fight and so should maintain a preference for Yield). Recall that if one monkey sequentially played Stag in the Assurance game first, Stag would also be a best response, which is what we observed. This implies that capuchins may be capable of playing a subgame perfect Nash equilibrium across a range of different games.

It is interesting that this only occurred in the asynchronous version, when they could see what their partner chose. This is consistent with their behavior in the assurance game and therefore provides additional support for the hypothesis that whatever mechanism the capuchin monkeys are using requires that they have information on their partner's decisions as they are making them; even learning the

\footnotetext{
${ }^{3}$ One monkey showed fewer than the expected number of runs on two sessions (Han: $p<0.001$ and $p=0.042$ ), one monkey showed more than the expected number of runs on one session (Luke: $p=0.016$ ), and one monkey showed a trend in that direction (Obi: $p=0.072$ ).
} 
information at the end of the trial (i.e., in the functionally simultaneous game, in which they saw their partner's choice after they made their own) is not sufficient. This inability to use information at a later time may be a substantial limitation on the ability of capuchin monkeys to coordinate in more complex situations. Although this seems like a liability for a group living, cooperative species, it may be due to their socio-ecology. As discussed in the introduction, capuchins typically live in small, cohesive groups that spend little time out of contact with one another (in contrast to the fission-fusion societies of chimpanzees and the very large group sizes of rhesus monkeys, which may be an order of magnitude larger than capuchin groups). There may have been little pressure for capuchins to evolve strategies that work in the absence of direct viewing of the others' choices or outcomes.

Unlike in the assurance game, in our conflict game the capuchins were only successful in the computerized task, but not the manual one. We suspect that the reason for this is largely based on the speed with which the task could progress in the computerized version, as compared to the more slowly paced manual version; the former may have made associations easier for the monkeys to make (e.g., rewards followed choices more rapidly and there was less time between trials). There were also additional trials per session, which has apparently helped capuchins learn the task in both this and the assurance game (in both this and the previous task, the order of play was confounded with the number of trials, making it impossible to rule out experience effects; Brosnan et al. 2012). Especially given the similarity in results across modalities in humans and in capuchins (in the assurance game), it is likely that the modality is not influencing behavior, but rather the number of and speed of trials.

Finally, it is interesting to speculate on how the animals coordinated which individual played Fight and which played Yield. Our original thought was that this was driven by dominance, with more dominant individuals playing Fight (which was higher-paying in the NE), but this was not born out by further testing. First, only 3 of 5 of the capuchins who chose Fight in Stage 1 were dominant to their partners, and more importantly, in Stage 2, all five of the pairs switched who played which icon, ruling out any possibility that the Fight choice was based on dominance. We hypothesize that the choice of strategy was stochastic, dependent upon who first happened to settle on Fight. That individual would do well, but when the second player switched to Fight, that second player would achieve a lower payoff than they had previously received with Yield, which encourages them to switch back to Yield.

Rhesus monkeys also found it difficult to play a Nash equilibrium. Although we had no a priori prediction about the rhesus' behavior, the success of the capuchins in playing the Nash equilibrium means that it is not surprising that the rhesus did so as well. In the assurance game, we hypothesized that the rhesus' success was based on a preference for the outcome that paid them the best, and we hypothesize that that was also the strategy that they used here, as this strategy would lead to Nash equilibrium play for the reasons outlined above. Note that they need not understand that they are matching/antimatching; they just need to show a robust preference for the outcome that, on average, pays them the best. Additionally, unlike in the assurance game, in which the rhesus' strategies were the same in the functionally simultaneous and asynchronous versions of the task, the rhesus monkeys in this case did much less well on the functionally simultaneous game than the asynchronous one. Unlike the capuchins, they did show evidence of playing a Nash equilibrium even in the simultaneous game, but their pattern of play was much less robust. ${ }^{4}$ These results reinforce the idea that rhesus are better able to find consistent outcomes even when they cannot see what their partner is doing, which we predicted was a result of their larger social groups (but it also indicates that there are limits to their ability).

\footnotetext{
${ }^{4}$ Of course, there is also an order effect here; pairs played the simultaneous game first and may have learned the strategy better by the time the played the asynchronous game.
} 
Unlike both species of nonhuman primate, the humans regularly played a Nash equilibrium and many pairs also alternated play between the two asymmetric Nash equilibria. Thus, unlike in the assurance game, in which some pairs of nonhuman primates showed the same pattern of Nash equilibrium play as did the humans, in the current anti-coordination tasks we see divergence, with humans playing differently in the repeated game. This may indicate a key difference between humans and other primates. One possibility is that humans strategize such social tasks differently than do the monkeys, allowing them to imagine play that is not apparent to the nonhuman primates. Future work should focus on where the limits of this difference lie. Interestingly, in both versions of the game we found a single pair that played the mutually destructive (Fight, Fight) outcome, which is another difference with nonhuman primates, who never consistently played (Fight, Fight). While we cannot draw conclusions from such a limited sample, the fact that this, too, was consistent across the two modalities - a small number of humans played (Hare, Hare) in the assurance game-more strongly indicates the possibility of a small subset of humans who get "stuck" in Pareto-dominated play. This is interesting for a future line of inquiry.

To our surprise, these results emphasize that both humans and non-human primates play Nash equilibria in our conflict game. This implies that, whatever the primates understand about the task, the ability to deal with conflict was of key importance. Indeed, although our work was designed to focus on the functional outcomes, and not the underlying mechanisms, a comparison of these results across species does provide some information on how the different species are solving the task. We find that capuchin monkeys, when they are able to solve the task, do so with very simple strategies. In the assurance game, they matched their partner's play, and in the current game, they settled on a preferred strategy and played it consistently, but failed to settle on the same strategy when presented with the same task, but novel tokens (i.e., subjects switched to the alternate strategy apparently stochastically). This is a key point; capuchins can successfully solve this task in some conditions, finding the NE at very high rates, apparently without sufficiently understanding the strategy to be able to apply it in other situations. Results such as these emphasize the diversity of ways that different outcomes may be reached and highlight the importance of not assuming mechanism from behavioral outcome.

Unlike previous work, this work is the first to show a clear split between humans and nonhuman primates in how they play a simple $2 \times 2$ game repeatedly. In previous work, all species were able to find the payoff dominant outcome, but in the current study, only humans alternate play of both asymmetric Nash equilibria in repeated play. This implies that humans are particularly well adapted to solve this task, which tells us something about the unique challenges faced during human evolution. Although again these results were not designed to inform us about the mechanisms underlying these decisions, we speculate that more advanced cognition is required in this alternating scenario. For example, subjects playing the alternating NE may need sufficient working memory to maintain several rounds of play in mind. Practically, the variability in these results indicate that this task, or related anti-coordination tasks, may be the most useful when teasing apart cognitive and behavioral differences between humans and other primates. 
Figure 1a: The Payoff Matrix for the Assurance Game (from Brosnan et al. 2011; 2012)

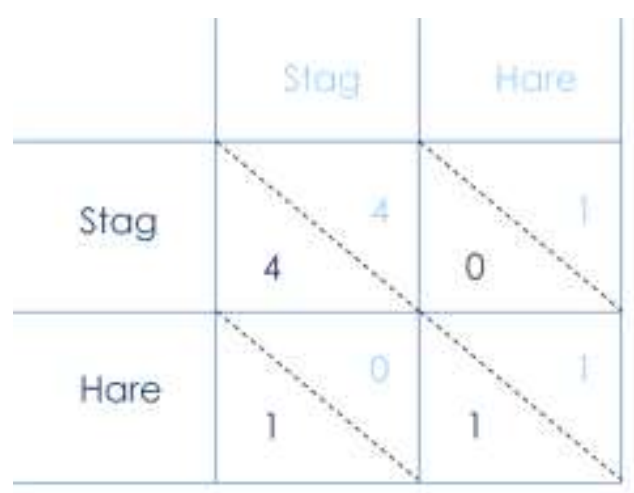

Figure 1b: The Payoff Matrix for Our Conflict Game

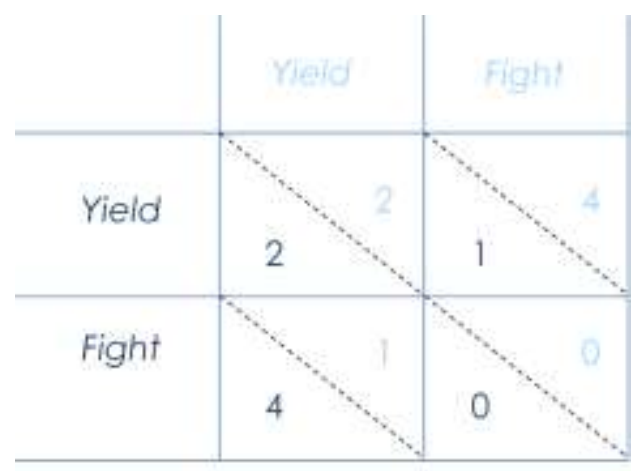


Figure 2: Proportion of Fight play by Capuchin Pair with Clear and Opaque Manual Tasks



Figure 3: Computer Testing Apparatus for Capuchins
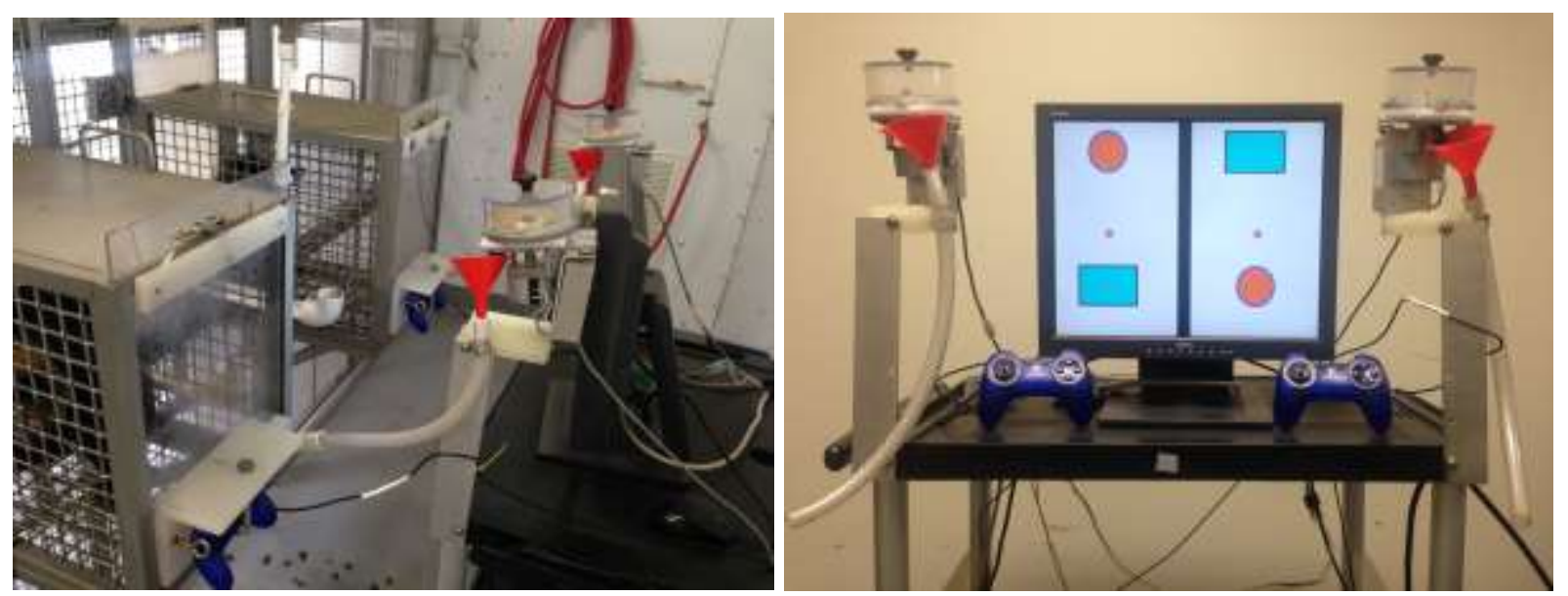
Figure 4: Proportion of Fight play by Capuchin Pair with Computer Tasks



Figure 5: Proportion of Fight play by Rhesus Pair with Computer Tasks

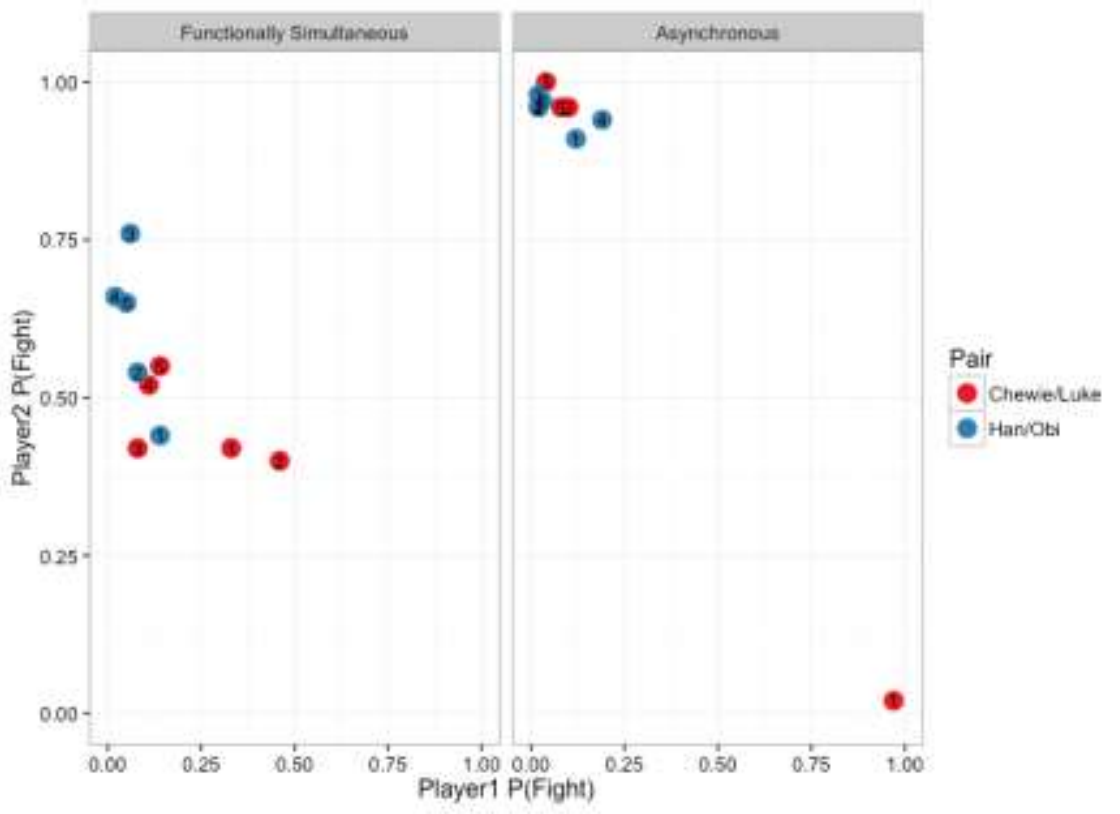




\section{References}

Addessi, Elsa, Lara Crescimbene, and Elisabetta Visalberghi. 2008. "Food and Quantity Token Discrimination in Capuchin Monkeys (Cebus Apella)." Animal Cognition 11(2):275-82. doi: 10.1007/s10071-007-0111-6.

Beran, Michael J., Theodore A. Evans, Katherine A. Leighty, Emily H. Harris, and Daniel F. Rice. 2008. "Summation and Quantity Judgments of Sequentially Presented Sets by Capuchin Monkeys (Cebus Apella)." American Journal Primatology 70(2):191-94. doi: 10.1002/ajp.20474.

Beran, Michael J., and John D. Smith. 2011. "Information Seeking by Rhesus Monkeys (Macaca Mulatta) and Capuchin Monkeys (Cebus Apella)." Cognition 120(1):90-105. doi: 10.1016/j.cognition.2011.02.016.

Beran, Michael J., John D. Smith, Mariana V. C. Coutinho, Justin J. Couchman, and Joseph Boomer. 2009. "The Psychological Organization of "Uncertainty" Responses and "Middle" Responses: A Dissociation in Capuchin Monkeys (Cebus Apella)." Journal of Experimental Psychology Animal Behavior Processes 35(3):371-81. doi: 10.1037/a0014626.

Boesch, C. (1994). Cooperative hunting in wild chimpanzees. Animal Behavior, 48: 653-667.

Brosnan, Sarah F., Michael J. Beran, Audrey E. Parrish, Sara A. Price, and Bart J. Wilson. 2013. "Comparative Approaches to Studying Strategy: Towards an Evolutionary Account of Primate Decision Making." Evolutionary Psychology 11(3):606-27. doi: 10.1177/147470491301100309.

Brosnan, Sarah F., Audrey E. Parrish, Michael J. Beran, Timothy Flemming, Lisa Heimbauer, Catherine F. Talbot, Susan P. Lambeth, Steven J. Schapiro, and Bart J. Wilson. 2011. "Responses to the Assurance Game in Monkeys, Apes, and Humans Using Equivalent Procedures." Proceedings of the National Academy of Sciences 108(8):3442-47. doi: 10.1073/pnas.1016269108.

Brosnan, S. F. (2010). What do capuchin monkeys tell us about cooperation? In D. R. Forsyth \& C. L. Hoyt (Eds.), For the Greater Good of All: Perspectives on Individualism, Society, and Leadership Perspectives on Individualism, Society, and Leadership (Vol. Jepson Studies in Leadership Series, pp. 11-28). New York, NY: Palgrave Macmillan Publishers.

Brosnan, Sarah F., Bart J. Wilson, and Michael J. Beran. 2012. "Old World Monkeys are More Similar to Humans Than New World Monkeys When Playing a Coordination Game." Proceedings of the Royal Society B: Biological Sciences 279(1733):1522-30. doi: 10.1098/rspb.2011.1781.

Chen, M. Keith, Venkat Lakshminarayanan, and Laurie R. Santos. 2006. "How Basic Are Behavioral Biases? Evidence from Capuchin Monkey Trading Behavior." Journal of Political Economy 114(3):517-37. doi: $10.1086 / 503550$.

Claidière, Nicolas, Andrew Whiten, Mary C. Mareno, Emily J. E. Messer, Sarah F. Brosnan, Lydia M. Hopper, Susan P. Lambeth, Steven J. Schapiro, and Nicola McGuigan. 2015. "Selective and Contagious Prosocial Resource Donation in Capuchin Monkeys, Chimpanzees and Humans." Scientific Reports 5:7631. doi: 10.1038/srep07631. 
Crowne, D. P., and D. Marlowe. 1960. "A New Scale of Social Desirability Independent of Psychopathology." Journal of Consulting Psychology, 24: 349-354.

Evans, Theodore A., Michael J. Beran, Emily H. Harris, and Daniel F. Rice. 2009. "Quantity Judgments of Sequentially Presented Food Items by Capuchin Monkeys (Cebus Apella)." Animal Cognition 12(1):97-105. doi: 10.1007/s10071-008-0174-z.

Gomes, Ursula. R., Daniel. M. A Pessoa, Carlos Tomaz, and Valdir F Pessoa. 2002. "Color Vision Perception in the Capuchin Monkey (Cebus Apella): A Re-Evaluation of Procedures Using Munsell Papers." Behavioural Brain Research 129(1-2):153-57. doi: 10.1016/S0166-4328(01)00335-7.

Higham, J., \& Maestripieri, D. 2010. "Revolutionary coalitions in male rhesus macaques." Behaviour 147(13): 1889-1908. https://doi.org/10.1163/000579510X539709

Horner, Victoria, J. Devyn Carter, Malini Suchak, and Frans B. M. de Waal. 2011. "Spontaneous Prosocial Choice by Chimpanzees." Proceedings of the National Academy of Sciences of the United States of America 108(33):13847-51. doi: 10.1073/pnas.1111088108.

House, Bailey R., Joan B. Silk, Susan P. Lambeth, and Steven J. Schapiro. 2014. "Task Design Influences Prosociality in Captive Chimpanzees (Pan Troglodytes)." PLOS ONE 9:e103422. doi: 10.1371/journal.pone.0103422.

Jacobs, Gerald. H. 2007. "New World Monkeys and Color." International Journal of Primatology 28(4):729-59. doi: 10.1007/s10764-007-9168-y.

Jensen, Keith, Josep Call, and Michael Tomasello. 2007. "Chimpanzees Are Rational Maximizers in an Ultimatum Game." Science 318:107-09. doi: 10.1126/science.1145850.

Jensen, Keith, Brian Hare, Josep Call, and Michael Tomasello. 2006. "What's in It for Me? Self-Regard Precludes Altruism and Spite in Chimpanzees." Proceedings of the Royal Society B: Biological Sciences 273(1589):1013-21. doi: 10.1098/rspb.2005.3417.

Kappeler, P. M., \& Van Schaik, C. P. (2005). Cooperation in Primates and Humans: Mechanisms and Evolution. Springer.

Maestripieri, D. (2007). Macachiavellian Intelligence. Chicago: University of Chicago Press.

Martin, Christopher F., Rahul Bhui, Peter Bossaerts, Tetsuro Matsuzawa, and Colin Camerer. 2014. "Chimpanzee Choice Rates in Competitive Games Match Equilibrium Game Theory Predictions." Science Reports 4:5182. doi: 10.1038/srep05182.

Maynard Smith, John. 1982. Evolution and the Theory of Games. Cambridge: Cambridge University Press. Maynard Smith, John, and George R. Price. 1973. "The Logic of Animal Conflict." Nature 246:15-18. doi: 10.1038/246015a0. 
Muller, M., \& Mitani, J. C. (2005). Conflict and cooperation in wild chimpanzees. In P. J. B. Slater, J. Rosenblatt, C. Snowdon, T. Roper, \& M. Naguib (Eds.), Advances in the Study of Behavior (pp. 275-331). New York: Elsevier.

Parrish, Audrey E., Sarah F. Brosnan, Bart J. Wilson, and Michael J. Beran. 2014. "Differential Responding by Rhesus Monkeys (Macaca Mulatta) and Humans (Homo Sapiens) to Variable Outcomes in the Assurance Game." Animal Behavior and Cognition 1(3):215-29. doi: 10.12966/abc.08.01.2014.

Pirta, R. S. 1983. "Cooperative Behaviour in Rhesus Monkeys Living in Urban and Forest Areas." Proceedings: Animal Sciences 92(3): 199-205. https://doi.org/10.1007/BF03186187

Santos, Laurie R., and Alexandra G. Rosati. 2015. "The Evolutionary Roots of Human Decision Making." Annual Review of Psychology 66:321-47. doi: 10.1146/annurev-psych-010814-015310.

Silk, Joan B., Sarah F. Brosnan, Jennifer Vonk, Joseph Henrich, Daniel J. Povinelli, Amanda S. Richardson, Susan P. Lambeth, Jenny Mascaro, and Steven J. Schapiro. 2005. "Chimpanzees are Indifferent to the Welfare of Unrelated Group Members." Nature 437(7063):1357-59. doi: 10.1038/nature04243.

Smith, Phillip, and Alan Silberberg. 2010. "Rational Maximizing by Humans (Homo Sapiens) in an Ultimatum Game." Animal Cognition 13(4):671-77. doi: 10.1007/s10071-010-0310-4.

Washburn, David A., and Duane M. Rumbaugh. 1991. "Ordinal Judgments of Numerical Symbols by Macaques (Macaca Mulatta)." Psychological Science 2(3):190-93. doi: 10.1111/j.14679280.1991.tb00130.x. 